\title{
An AGAMOUS-like factor is associated with the origin of two domesticated varieties in Cymbidium sinense (Orchidaceae)
}

\author{
Shihao Su ${ }^{1,2}$, Xiaoyu Shao ${ }^{1}$, Changfa Zhu' ${ }^{1}$, Jiayin Xu' ${ }^{1}$, Yuhuan Tang ${ }^{1}$, Da Luo ${ }^{1}$ and Xia Huang ${ }^{1}$
}

\begin{abstract}
Cymbidium has been artificially domesticated for centuries in Asia, which produced numerous cultivated varieties. Flowers with stamenoid tepals or those with multiple tepals have been found in different species of Cymbidium; however, the molecular basis controlling the formation of these phenotypes is still largely unknown. Previous work demonstrated that AGAMOUS/AG lineage MADS genes function in floral meristem determinacy as well as in reproductive organs development in both dicots and monocots, indicating a possible relationship with the origin of two flower varieties in Cymbidium. Here, we characterized and analyzed two AG lineage paralogues, CSAG1 and CSAG2, from Cymbidium sinense, both of which were highly expressed in the gynostemium column of a standard $C$. sinense. Interestingly, we detected ectopic expression of CSAG1 rather than CSAG2 in all floral organs of a stamenoid-tepal variety and significant down-regulation of CSAG1 in a variety with multiple tepals. Over-expression of CSAG1 in wild type Arabidopsis resulted in petal-to-stamen homeotic conversion, suggesting a conserved C-function of CsAG1 in the development of Cymbidium flower. Altogether, our results supported a hypothesis that disruption of a single AG-like factor would be associated with the formation of two domesticated varieties in C. sinense.
\end{abstract}

\section{Introduction}

The Cymbidium spp. have been cultivated for more than ten centuries in Asia, including China, Japan, Korea, and many other places ${ }^{1,2}$. After hundreds of years of domestication, numerous varieties with diversification in inflorescence architecture, leaf or flower color, fragrance as well as tepal shape have been produced. Among these varieties, flowers with stamenoid tepals or those with multiple tepals widely exist in different Cymbidium species such as C. goeringii, C. faberi, and C. sinense, which were documented in an ancient Chinese Orchidology book named "Nan-Zhong-You-Fang-Lu" in around 1412 Common Era.

\footnotetext{
Correspondence: Xia Huang (huangxia@mail.sysu.edu.cn)

${ }^{1}$ State Key Laboratory of Biocontrol and Guangdong Key Laboratory of Plant Resources, School of Life Sciences, Sun Yat-sen University, Guangzhou 510275, China

${ }^{2}$ Institute of Transformative Bio-Molecules (WPI-ITbM), Nagoya University, Furocho, Chikusa-ku, Nagoya 464-8601 Aichi, Japan
}

These authors are contributed equally: Shihao Su and Xiaoyu Shao
A standard C. sinense flower (CsWT) possesses three distinct whorls of floral organs (Fig. 1a). The outermost whorl consists of three long outer tepals, also known as sepals; the second whorl consists of three shorter inner tepals, also known as petals, with the dorsal one differentiated into a spotted showy lip; the innermost whorl is the reproductive structure called gynostemium or column, where both male and female reproductive organs are fused together into a single column (Fig. 1b). Due to the highly specialized floral organs and numbers of cultivated varieties, $C$. sinense becomes an ideal material to study the origin of particular floral forms ${ }^{3}$.

MADS-box genes containing a highly conserved $M$ domain have been widely recruited in the flower developmental processes, among which the AGAMOUS/AG lineage is involved in floral meristem determinacy and confers the identity of reproductive floral organs including stamens and carpels ${ }^{4-9}$. Phylogenetic analyses of different $A G$-like sequences demonstrated that gene

\section{(c) The Author(s) 2018}

(c) (i) Open Access This article is licensed under a Creative Commons Attribution 4.0 International License, which permits use, sharing, adaptation, distribution and reproduction cc) in any medium or format, as long as you give appropriate credit to the original author(s) and the source, provide a link to the Creative Commons license, and indicate if changes were made. The images or other third party material in this article are included in the article's Creative Commons license, unless indicated otherwise in a credit line to the material. If material is not included in the article's Creative Commons license and your intended use is not permitted by statutory regulation or exceeds the permitted use, you will need to obtain permission directly from the copyright holder. To view a copy of this license, visit http://creativecommons.org/licenses/by/4.0/. 


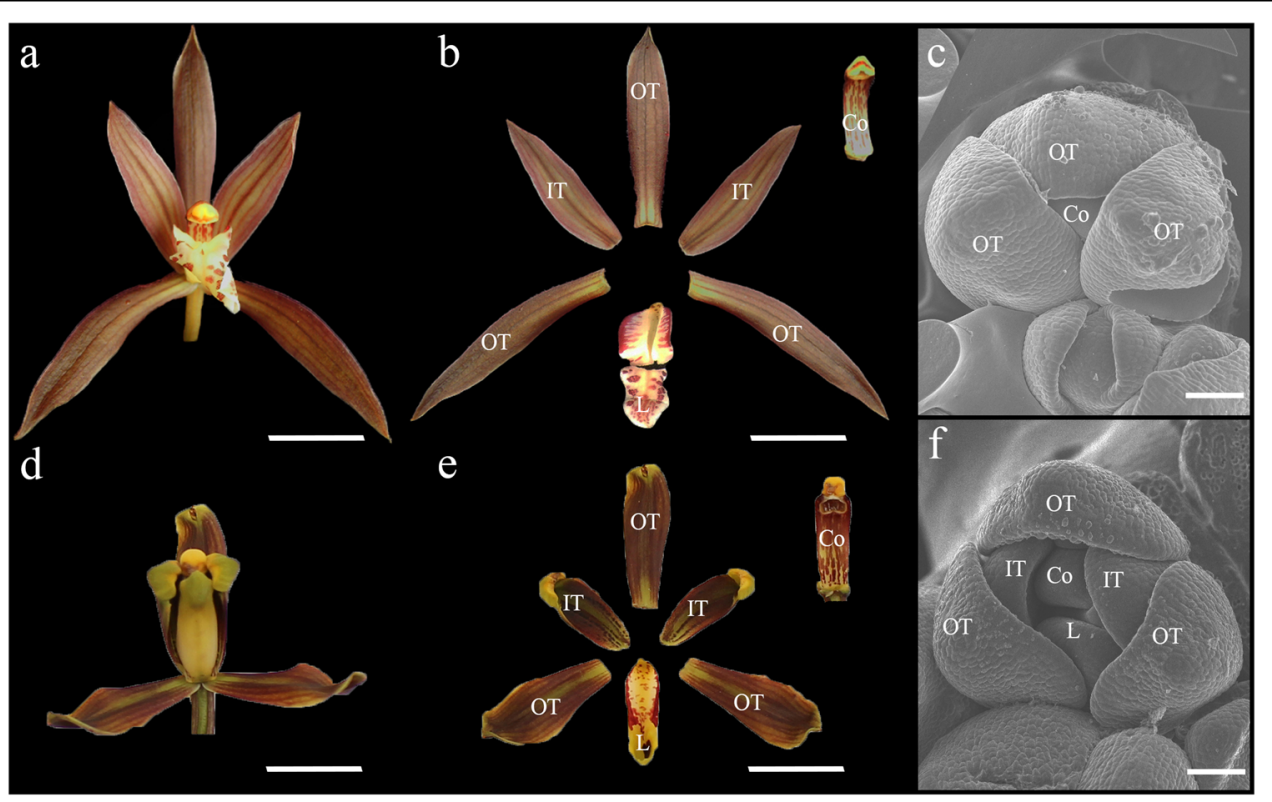

Fig. 1 Floral phenotypes of the standard and stamenoid-tepal Cymbidium sinense. A mature standard (a) and stamenoid-tepal (d) C. sinense flower; a dissected standard (b) and stamenoid-tepal (e) C. sinense flower; OT, outer tepal; IT, inner tepal; L, lip; Co, column; bar = 10 mm. Scanning electron microscopic photographs of a developing standard (c) and stamenoid-tepal (f) flower; bar $=100 \mu \mathrm{m}$

duplications occurred extensively during the evolution of this subfamily ${ }^{10}$. In core eudicots, the AG lineage can be further divided into two sub-clades including the euAG and PLENA/PLE. Within monocots and other basal eudicots, the evolutionary scenario of AG lineage factors is still obscure, although multiple gene duplication events have been observed ${ }^{6,10}$.

The function of AG lineage factors has been very well characterized in the core eudicots ${ }^{7-13}$. In Arabidopsis thaliana, mutation in the euAG sub-clade member $A G$ exhibits loss of stamen and carpel identity, with defects in floral meristem determinacy that result in the development of another flower in place of the carpel ${ }^{9,13}$. Further, AG interacts antagonistically with APETALA2/AP2, another floral homeotic gene regulating normal development of the first sepal whorl and the second petal whorl $^{8,9}$. AP2 restricts the expression of $A G$ gene in the two inner whorls, or counteracts AG activity in the center of flower ${ }^{8,9,14}$. In snapdragon (Antirrhinum majus), mutation in euAG sub-clade FARINELLI/FAR produces normal flowers with partially male-sterile ${ }^{12}$; while in petunia (Petunia hybrida), down regulation of euAG subclade gene PETUNIA MADS BOX GENE3/PMADS3 results in mild abnormalities in reproductive organ development ${ }^{11}$.

The PLE sub-clade $A G$ factors work redundantly with the euAG sub-clade members. Two PLE sub-clade genes from Arabidopsis, SHATTERPROOF/SHP1 and SHATTERPROOF2/SHP2, exhibit partly redundant function to $A G$, responsible for stamen and carpel development ${ }^{15}$. In snapdragon, reproductive organs of ple-1 (PLE sub-clade) mutant are converted into perianth organs, showing severely developmental defects when compared to the far (euAG sub-clade) mutant ${ }^{12}$. Genetic analysis suggests that $F A R$ negatively regulates the expression of $P L E^{12,16}$. In petunia, PLE sub-clade FLORAL BINDING PROTEIN6/ FBP6 and euAG sub-clade PMADS3 have largely overlapping in function, determining reproductive organ identity as well as floral determinacy ${ }^{11}$.

In grasses, such as rice (Oryza sativa) and maize (Zea mays), the $A G$-like members have also been analyzed. In rice, two members, OsMADS3 and OsMADS58, belong to the AG lineage. In the knockout line of OsMADS3, stamens transform into lodicules and ectopic lodicules develop in the second whorl of floral organs ${ }^{5}$. In the osmads 3 osmads 58 double mutant, the reproductive organ identity is completely lost together with the loss of floral meristem determinacy, and massive lodicules-like structures appear in the third and fourth whorls ${ }^{17}$. In maize, there are four members in AG lineage, including ZAG1, ZAG2, ZMM2 and ZMM23. ZAG1 is highly expressed in stamen and carpel primordia; however, those mutants show a loss of floral meristem determinacy, rather than serious defects in reproductive organ identity ${ }^{4,18}$. The different expression pattern of these genes favors subfunctionalized behaviors of maize AG-like factors in regulating stamen, tassel, and carpel development of male and female flowers ${ }^{4,6,18-20}$.

Several AG lineage factors have been isolated from Orchidaceae species ${ }^{21-26}$. In Dendrobium crumenatum, 


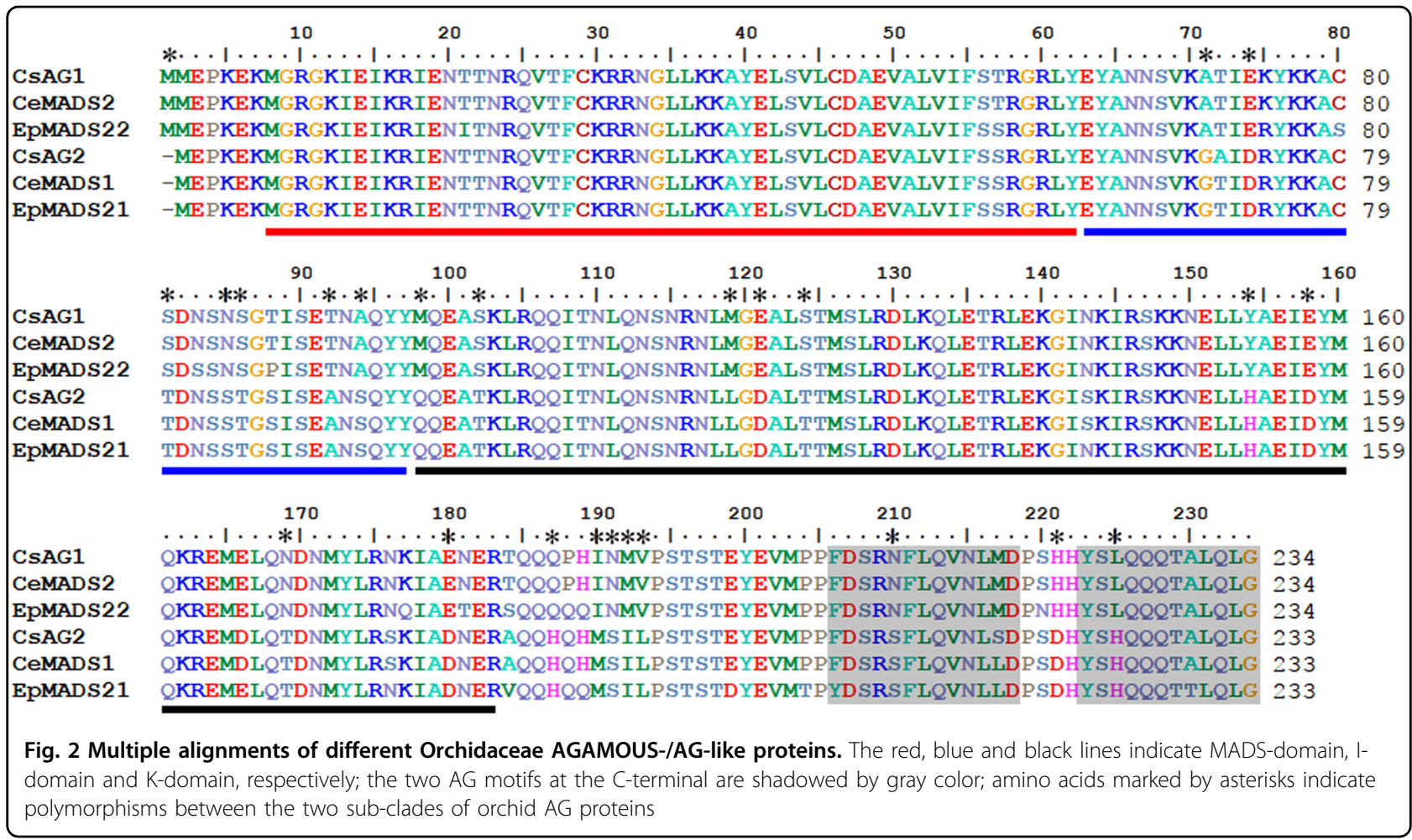

the putative $\mathrm{C}$ function gene $\mathrm{DcOAG1}$ is highly expressed in all the floral organs, which leads to ap2-like phenotypes when ectopically expressed in Arabidopsis ${ }^{23}$. In the orchid Erycina pusilla, three AG lineage factors EpMADS20/21/ 22 are all strongly expressed in the column whorl indicating possible functional redundancy in male and female reproductive organ development ${ }^{23}$. In a more closed relative of $C$. sinense, $C$. ensifolium, duplicated AG lineage genes denoted CeMADS1/2 have been characterized ${ }^{24}$. In the multitepal mutant, whose column has been centripetally replaced by numerous tepal-like structures, the normal expression of CeMADS1 rather than CeMADS2 is disrupted $^{24}$. Interestingly, in the gylp mutant from Phalaenopsis equestris, whose two inner tepals change into gynostemium-like structures, an $A G$ lineage gene, PeMADS1, is ectopically expressed in the gynostemiumlike tepals ${ }^{26}$. All these findings give a strong indication that defects in AG-like factors would have occurred in the origin of the stamenoid-tepal and multi-tepal varieties in C. sinense.

In this study, we have isolated two AG lineage paralogs from C. sinense, and phylogenetically analyzed the evolution of AG lineage factors in Orchidaceae. We observed the floral developmental abnormalities between the standard and stamenoid-tepal variety in the early floral developmental stage. By detecting the expression patterns of these factors, we found that CsAG1 but not CsAG2 is ectopically expressed in all floral organs of the stamenoidtepal variety, while extremely low expression of CsAG1 could be detected in a multi-tepal variety. We further ectopically expressed CsAG1 in wild type Arabidopsis, and observed petal-to-stamen homeotic conversion in several independent transgenic lines, supporting a conserved Cfunction of CsAG1 in Cymbidium flower development. Our results support not only the occurrence of a duplication event during the diversification of Orchidaceae AG lineage, but also a possible link between CsAG1 and the origin of different floral varieties in $C$. sinense.

\section{Results}

Flower comparison between the standard and stamenoidtepal $C$. sinense

We dissected a mature flower from the standard and stamenoid-tepal C. sinense, respectively (Fig. 1b, e). The variety with stamenoid tepals in $C$. sinense named "LingNan-Da-Mei" (Csm, Fig. 1d). "Mei" literally in Chinese describes plum-blossom-shaped flower, whose outer tepals become shorter compared with those on the standard C. sinense (Fig. 1e). In the standard C. sinense, the two inner tepals stretch outward naturally, while the lip forms a coil decorated with different patterns of pigments (Fig. 1b). However, in the stamenoid-tepal variety, those abnormal areas on the inner tepals and lip mimic pollinium structures of the column, forming three inward pockets bending toward the stamen (Fig. 1e). The distal margins of three outer tepals in the stamenoid-tepal variety also become oval and curved compared with the standard C. sinense (Fig. 1b, e). We observed the early 
flower developmental process of the two varieties using scanning electron microscopy (SEM). Although the initiation of floral organ primordia is normal in the stamenoid-tepal variety compared with the standard (data not shown), the same does not occur in later developmental processes. The morphology of the tepals in the stamenoid-tepal variety becomes abnormal, with the whole floral meristem forming an equilateral triangle shape and the top regions of the outer tepals becoming curved, not being able to fully cover the inner floral organs (Fig. 1c, f).

\section{Isolation and phylogeny of AGAMOUS lineage factors from C. sinense}

Since genes from the AGAMOUS lineage play a vital role in the determination of plant reproductive organ development, we isolated the cDNA of two $A G$-like factors from the inflorescence of the standard $C$. sinense. CsAG1 and CsAG2 encode two putative MADS proteins with 234 and 233 amino acids, respectively (Fig. 2). We downloaded different published sequences, which belongs to AG lineage, from eudicot Arabidopsis, the grass family species rice and maize, other monocots species including Ananas comosus, Musa acuminata, Asparagus virgatus, Hyacinthus orientalis as well as sequences of different Orchidaceae species. Phylogenetic analysis using maximum-Likelihood method showed that AG lineage factors have undergone multiple duplication events during the diversification of angiosperms (Fig. 3). Consistent with previous studies, the duplication events occurred in eudicots were independent with those happened in monocots (Fig. 3). Similarly, independent duplication events were observed in Poaceae, M. acuminata and Orchidaceae evolutionary processes, and there have been two different sub-clades within Orchidaceae AG lineage named OrchidAG1 and OrchidAG2 (Fig. 3). Multiple alignments of the two sub-clades of AG proteins from Orchidaceae revealed three conserved protein domains including MADS-domain, I-domain and K-domain, together with two AG motifs in the C-terminal end (Fig. 2). In addition, we have also detected 25 single amino acid polymorphisms between the two sub-clades of orchid AG proteins (Fig. 2). These results indicated that diverged AG-like factors may have been involved in the regulation of orchid flower development.

\section{Expression of AGAMOUS lineage factors in standard and stamenoid-tepal $C$. sinense}

We then studied the expression patterns of these two AG lineage genes in different floral organs. Flower buds in different developmental stages were dissected into four parts, including three outer tepals (OT), two inner tepals (IT), a lip (L), and finally, one column (Co). To detect whether the expression of these factors was

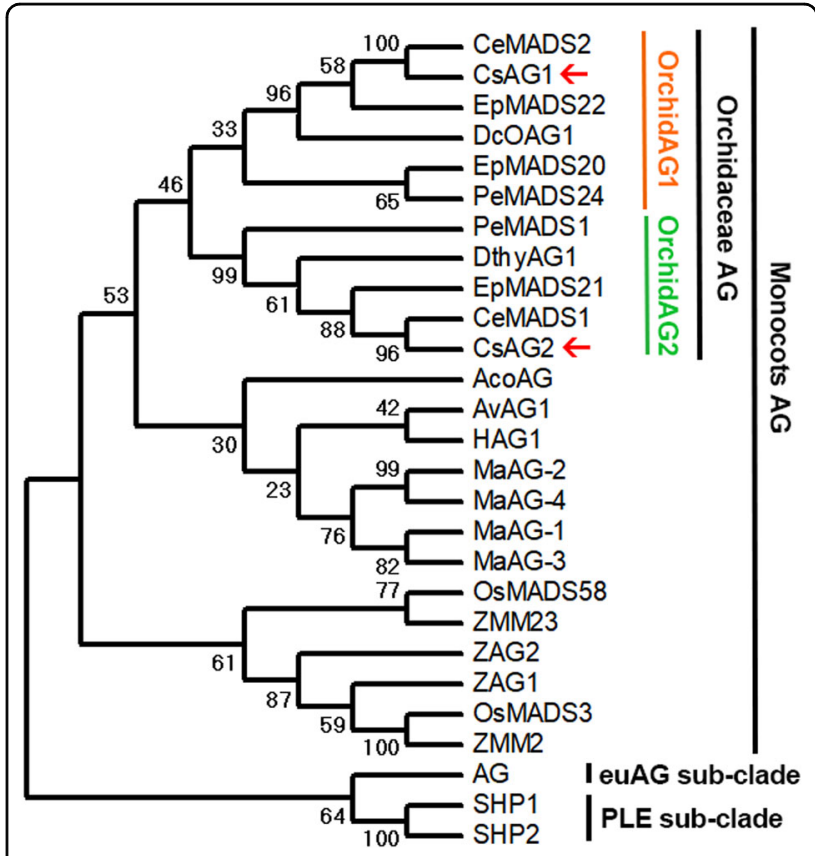

Fig. 3 Maximum-likelihood phylogeny tree of AGAMOUS-/AGlike proteins. Proteins from Arabidopsis were selected as outgroups; 500 replicates of bootstrap values are marked on each node; the red arrows indicate two AG paralogues isolated from Cymbidium sinense

stage-dependent, we preliminarily examined their expression among three different developing flowers with the bud length of $5 \mathrm{~mm}$ (f2), $10 \mathrm{~mm}$ (f3), and $15 \mathrm{~mm}$ (f4). Although the expression levels of these genes varied in different developmental stages, the overall expression patterns were consistent with the three stages showing high expression in the third column whorl (Fig. 4a, S1). Thus, in later qRT-PCR experiments, we chose f3 stage flowers as materials.

We checked the expression levels of two $A G$ paralogues in the stamenoid-tepal variety (Fig. 4b). The results showed that the expression pattern of CsAG2 was normal compared with the standard Cymbidium flower, in which this gene was specifically expressed in the column tissues. Interestingly, ectopic expression of CsAG1 in outer tepals, inner tepals and lip was detected, being consistent with the stamenoid structures on these floral organs (Fig. 4).

We further performed RNA in situ hybridization to check the spatial expression of CsAG1 (Fig. 5). In the standard Cymbidium flower, strong signals could be detected in the stamens and carpels but not the whole column when using anti-sense probe of CsAG1 (Fig. 5a). The signal was specific since it could not be detected when we used the sense probe (Fig. 5d). Similar with the qRT-PCR assays, ectopic expression of CsAG1 could be observed in the outer tepal with homeotic conversion in the stamenoid-tepal variety (Fig. 5b). We also detected 


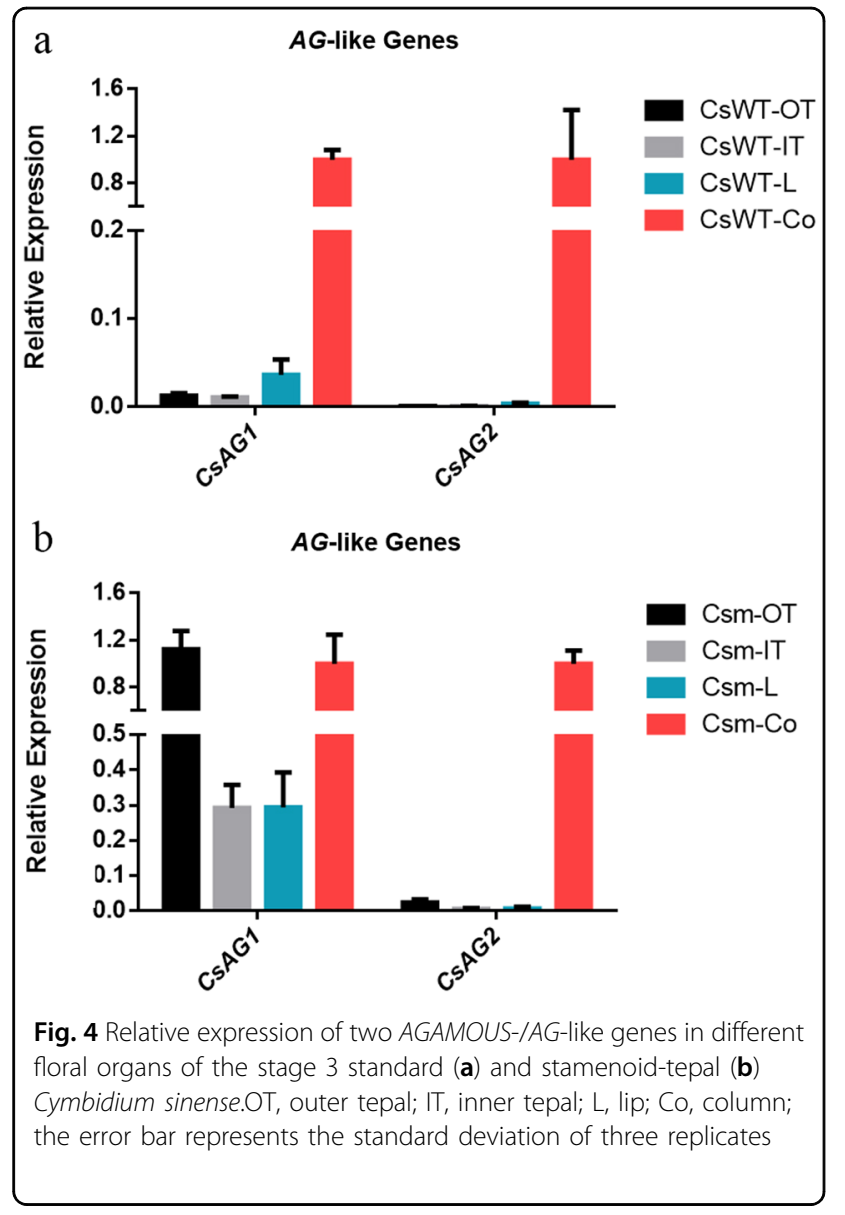

weak signal in the inner tepal and lip margin from continuous sections of the in situ hybridization assays, which was consistent with the qRT-PCR experiments (Fig. 5c).

\section{Characterization of another $C$. sinense variety with multiple tepals}

In addition, we have collected a variety named Da-TunQi-Lin (Csql) with defects in floral meristem determinacy (Fig. 6). "Qi-Lin", literally in Chinese, refers to a mythical chimerical creature with fire surrounding its body. Different types of flowers grow along the inflorescence (Fig. 6a). In the severe type, numerous tepals develop in the place of the column (Fig. 6b). In the weak type, several additional tepals surround the abnormal column (Fig. 6c). Remarkably, the identity of three outer tepals, two inner tepals and the lip is not affected in the multi-tepal variety, which is similar with the $a g$-like mutants in eudicots (Fig. 6b, c).

Thus, we checked the expression of $A G$ lineage genes in the multi-tepal variety. Since different degrees of abnormal flowers develop in one plant, we pooled the additional tepals or the abnormal columns, and designated as multitepal (MT) to make comparison with the column (Co) tissue in the standard flower. qRT-PCR analysis unraveled that the expression of CsAG1 and CsAG2 were all downregulated in the MT tissue of the multi-tepal variety (Fig. 7). Specifically, the expression of CsAG1 became extremely low, indicating strong association with the multi-tepal phenotype.

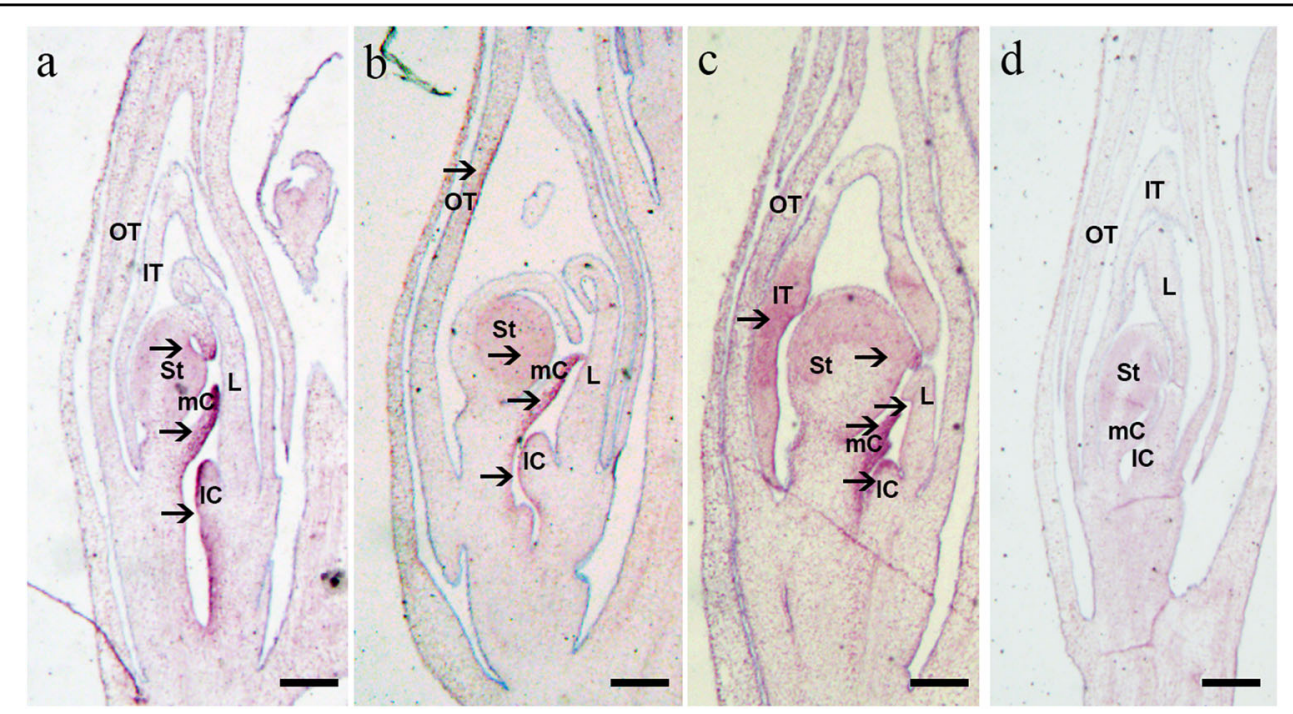

Fig. 5 RNA in situ hybridization detected by CSAGI antisense probe in standard (a) and stamenoid-tepal (b, c) Cymbidium sinense; dark regions marked by black arrows indicate strong signals; (d) is a negative control using the sense probe of CSAG1 in a standard flower; OT, outer tepal; IT, inner tepal; L, lip; St, stamen; mC, median carpel; IC, lateral carpel; bar $=100 \mu \mathrm{m}$ 


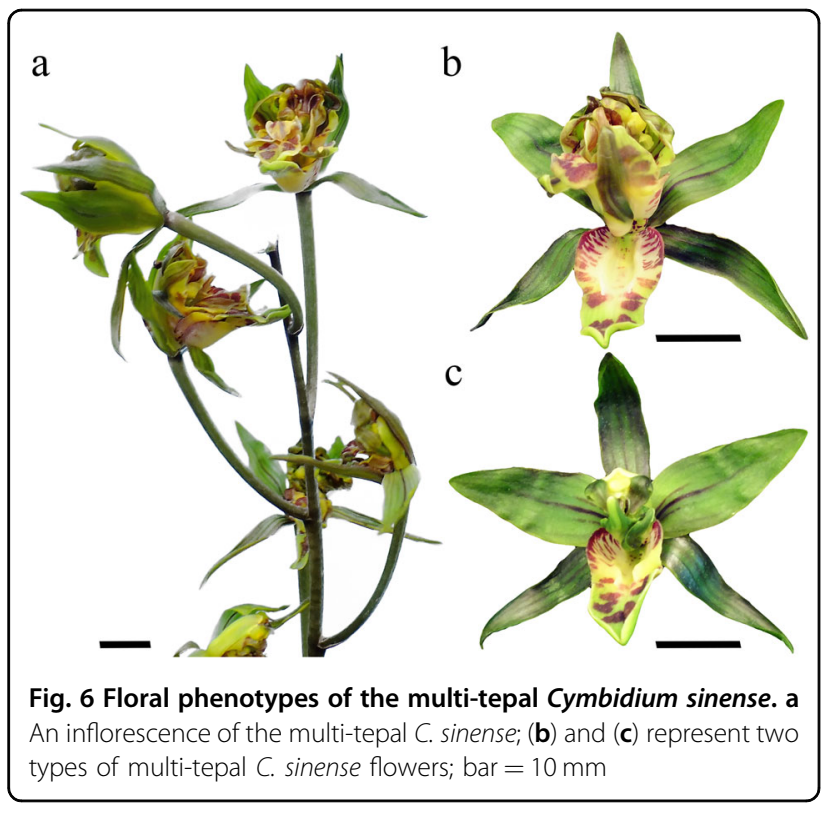

\section{Phenotypes of 35S:CsAG1 in Arabidopsis}

Based on the association between phenotype and expression, we inferred a major $\mathrm{C}$ function in Cymbidium flower that CsAG1 conferred. To verify this hypothesis, we ectopically expressed CsAG1 in Arabidopsis (Fig. 8). In three independent 35 S:CsAG1 transgenic lines, normal petal identity was disrupted (Fig. 8). Homeotic conversion of petal to stamenoid structure could be detected at the petal margin, which was similar as previous works reported in the eudicots (Fig. 8). These results indicated CsAG1 would possible be a key component with $\mathrm{C}$ class function responsible for the orchid reproductive organ development.

\section{Discussion}

Studies in the $A G$ lineage genes have been widely conducted in different plant lineages, drawing the conclusion that this gene lineage is associated with floral meristem determinacy and reproductive organ development ${ }^{5,7,9,11,12,18,20}$. AG lineage has undergone multiple events of duplication in both monocots and eudicots, leading to possible subfunctionalization ${ }^{6,10,27,28}$. The evolutionary scenario of $A G$ lineage is clear in core eudicots with one ancient duplication event in lower eudicots and subsequent production of two sub-clades, including euAG and $P L E^{6,10}$. Recent duplication events have also been observed in each lineage, as in the case of two AG-like genes from Arabidopsis, SHP1 and SHP2, which evolved distinct function from $A G$ after a duplication event ${ }^{29}$.

In monocots, independent duplication events in $A G$ lineage have been observed ${ }^{6,10,27,28}$. In the grass family (Poaceae) before the divergence of maize, rye, wheat and rice, a gene duplication event happened producing two

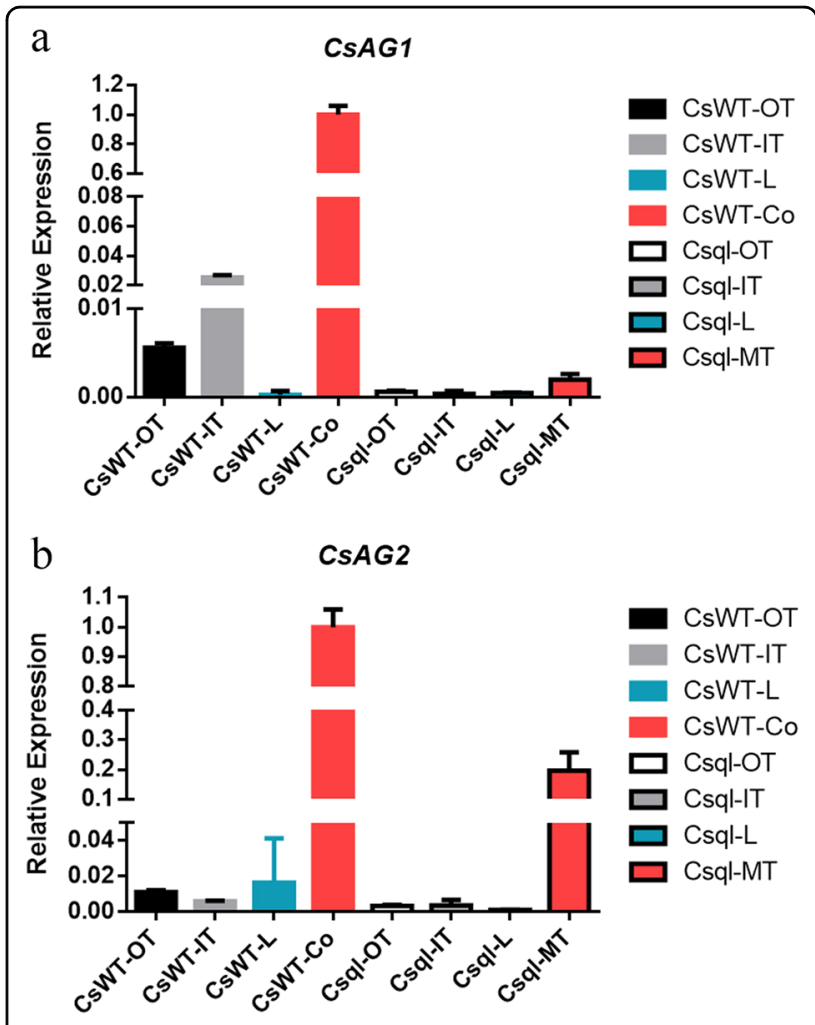

Fig. 7 Relative expression of two AGAMOUS-/AG-like genes, CSAG1 (a) and CSAG2 (b), in different floral organs of the stage 3 standard (CsWT) and multi-tepal (Csql) Cymbidium sinense.OT outer tepal, IT inner tepal, L lip, Co column, MT multiple inner organs, the error bar represents the standard deviation of three replicates

paralogous $A G$ lineages defined as $Z A G 1$ and $Z M M 2^{10}$. After an allotetraploid event occurred in maize approximately 11.4 Mya, two additional maize $A G$ paralogous genes produced, designated as $Z A G 2$ and $Z M M 23^{10,30}$. Outside Poaceae, for example, predating the divergence of the Zingiberales, there have been at least two clear subclades of the $A G$ gene resulted from a single duplication event named ZinAG-1 and ZinAG-2 ${ }^{27}$. It is of interest that the expression patterns of the two sub-clades $A G$ genes vary in different Zingiberales species, indicating possible mechanisms for the evolution of androecial petaloidy in Zingiberales $^{27}$.

In Orchidaceae, there have been two different subclades of $A G$ lineage factors resulted from a duplication event predating the divergence of Orchidaceae species (Fig. 3). Both of the two AG-like factors were highly expressed in columns of a standard Cymbidium flower (Fig. S1), and this is consistent as previous work reported in Erycina pusilla, indicating functional redundancy of $A G$ lineage factors in Orchidaceae ${ }^{27}$. In this study, we detected the spatial expression pattern of CsAG1 using RNA in situ hybridization, providing a more detailed pattern in top stamen margin, median carpel as well as the 


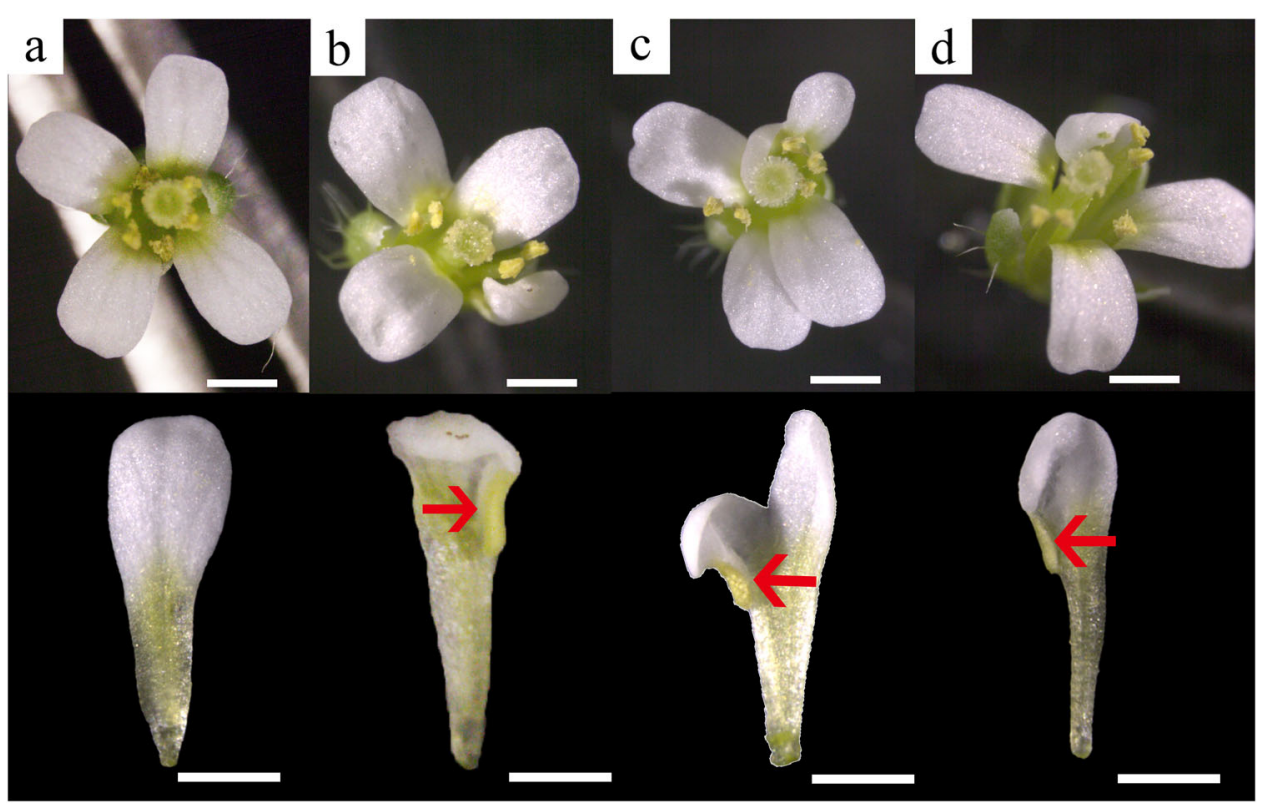

Fig. 8 Floral phenotypes of Col-0 (a) and three independent lines of 35S:CSAG1 transgenic Arabidopsis (b-d); For each genotype, the upper panel shows an intact flower and the lower panel exhibits a corresponding normal (Col-0) / stamenoid (35S:CSAG1) petal; red arrows indicate fused pollen sacs on the stamenoid petals; bar $=2 \mathrm{~mm}$.

stigma lobe (Fig. 5). We neither detected any signal of CsAG1 in the floral meristem, nor in the early flower developmental stages, different from the expression of an OrchidAG2 sub-clade member PeMADS1 in Phalaenopsis equestris, which is abundant in the whole floral meristem $^{26}$. Future work using in situ hybridization assays will allow a precise examination of the expression patterns of different $A G$-like factors, leading to a better understanding under subfunctionalization among these different paralogues.

In the flowers of stamenoid-tepal C. sinense variety, although homeotic conversion occurred on each tepal's marginal regions, the inner floral organs show a severe phenotype comparing to the outer floral organs, despite the fact that higher ectopic expression of CsAG1 was detected in the outer tepals when compared with the inner tepals and lips (Fig. 1). One possible explanation is the formation of different protein complexes. In Arabidopsis, MADS genes determine the floral organ identity through a combinatory way named the ABCDE model $^{31,32}$. Different MADS proteins can form different quaternary protein complexes and bind to the DNA region called CArG-box to regulate the expression of their downstream genes targets ${ }^{31,32}$. In Orchidaceae, previous studies reported that two duplication events happened in the AP3 sub-clade MADS factors, leading to divergence in expression patterns and probably resulting to functional diversification $^{33-35}$. The AP3-3 and AP3-4 sub-clades members are highly expressed in the two inner floral organ whorls, while the transcripts of AP3-1 and AP3-2 are abundant in the outer tepals and inner tepals, which may result in the formation of different MADS complexes in the stamenoid-tepal C. sinense variety ${ }^{33-35}$.

In Cymbidium ensifolium, an Orchidaceae AG2 sub-clade member, CeMADS1, is not expressed in multitepal flower buds $^{24}$. Unlike the multi-tepal variety of $C$. sinense in this study, the multitepal mutants of $C$. ensifolium completely lose the column, together with abnormalities developed in the inner tepals and the $\operatorname{lip}^{24}$. Since different Cymbidium species have been bred independently, it is possible that different mutations occurred during the generation of these varieties, which favor potential functional divergence within the two clades of AG proteins. A previous study found that complex autoregulatory networks of MADS proteins exist during the floral development of Arabidopsis ${ }^{36}$. Another possible explanation for generation of the multi-tepal phenotype in $C$. sinense would be a combinatory manner of two $A G$-like genes, since both of which were down-regulated in the multi-tepal variety.

Column, also known as gynostemium, is a very exquisite structure which has attracted many naturalists since 19th century. Charles Darwin noticed that the specialized structures orchid flower reflects the beauty of the adaptations ${ }^{37}$. A mature column consists of anther, lateral and ventral gynostemium appendages, ovary and calyculus, rostellum and stigma lobes, column-part as well as column-foot ${ }^{38-40}$. Although the morphologically developmental processes of the column have been well described, the underlying molecular mechanisms controlling the structure differentiation remain unclear. Since 
numerous varieties exist in the Cymbidium genus, it would be a choice to use these varieties to study the dark matter behind the orchid flower. Due to the developmental novelties in Orchidaceae flower, it will be exciting to utilize a model system, such as Erycina pusilla, for functional studies in the future ${ }^{41}$.

\section{Materials and Methods}

\section{Plant materials and nucleic acid extraction}

All the cultivated Cymbidium varieties analyzed in this paper were kept in the greenhouse of National Orchid Conservation Center of China and Orchid Conservation and Research Center of Shenzhen, Shenzhen, China. The Arabidopsis were grown in growth chambers at $22^{\circ} \mathrm{C}$ under a $16 \mathrm{~h}$ of light $/ 8 \mathrm{~h}$ dark with $70-80 \%$ relative humidity. The Arabidopsis and Cymbidium genomic DNA was extracted from juvenile leaves by DNA extraction solution containing $2 \%$ CTAB. Plant total RNA was extracted from different tissues using Plant RNA Kit (Omega Bio-Tek, Guangzhou, China).

\section{Scanning electron microscopy}

Five centimeter inflorescences were collected for SEM observation. To generate the epoxy replica, the unnecessary tissues were quickly removed and the inflorescences were dissected. The 1st type of impression material (Coltene Ltd. PRESIDENT light body, Art. No. 4667, Switzerland) was daubed onto the surface of dissected inflorescences, and the epoxy mold was fixed upside down on the 2nd type of vinyl polysiloxane impression material (Imprint $^{\text {tw }}$ II Garant, 3 M ESPE, U. S. A.). To fill the mold with the 3rd type of epoxy adhesive (Devcon Ltd. 2-Ton Epoxy, Ireland), the inflorescences were removed thoroughly under a stereomicroscope and were kept into $37^{\circ} \mathrm{C}$ incubator overnight. The epoxy replicas were sputtered with gold and observed under a JEOL JSM 6360LV Scanning Electron Microscope. The photo contrast was adjusted by using Adobe Photoshop CS6 (Adobe, San Jose, CA, USA).

\section{Molecular cloning and phylogenetic analysis}

To get completed sequences of $A G$-like genes, gene specific primers were designed in $5^{\prime}-13^{\prime}$-UTR regions and amplification were carried out using genomic DNA and 5 $\mathrm{cm}$ inflorescence cDNA as templates, respectively. PCR products were cloned into pMD19-T vector (Takara) before sequencing. The primers sequences used for molecular cloning were listed in the Supporting Information (Table S1). To perform phylogenetic analysis, putative coding region of each gene was predicted by NCBI ORFFinder (https://www.ncbi.nlm.nih.gov/orffinder/). The nucleotide sequences were further translated in amino acid sequences prior to multiple alignment using MEGA6, and the aligned sequences were further processed to generate Maximun Likelihood tree under 500 of bootstrap replicates ${ }^{42}$.

\section{Quantitative RT-PCR}

A total of $0.5 \mu \mathrm{g}$ RNA was reversely transcribed and the PCR assays were performed as we previously reported ${ }^{43}$. All the data were normalized against the expression of reference gene ACTIN, as previously described ${ }^{44}$. The transcript levels for these genes were summarized from three replicates. All the primers sequences used in qPCR were listed in the Supporting Information.

\section{RNA in situ hybridization}

Five centimeter inflorescences were collected for in situ hybridization. After removing unnecessary tissues and large flower buds, the dissected inflorescences were fixed overnight in $4 \%(\mathrm{wt} / \mathrm{vol})$ paraformaldehyde buffer $(\mathrm{pH}$ 7.0), which were further embedded with Paraplast (SigmaAldrich China, Shanghai, China). The digoxigenin-labeled probes were made and the hybridization processes were carried out as described ${ }^{45}$. All the primers sequences used in RNA in situ hybridization were listed in the Supporting Information.

\section{Plant transformation}

The coding region of CsAG1 was cloned and inserted into the multiple clone site of a binary vector pCAMBIA1302, which was further transformed into the Agrobacterium tumefaciens strains. The Agrobacteriummediated plant transformation was carried out using the floral dipping method as previously described ${ }^{46}$. Seeds of the 35S:CsAG1 transgenic plants were germinated and selected on Murashige and Skoog (MS) culture media containing Hygromycin B (Roche, Shanghai, China).

\section{Accession numbers}

The accession numbers of sequences used for phylogeny from GenBank (https://www.ncbi.nlm.nih.gov/genbank/) or Phytozome 12 (https://phytozome.jgi.doe.gov/) are as follows: AG (X53579); SHP1 (M55550); SHP2 (M55553); OsMADS3 (L37528); OsMADS58 (AB232157); ZAG1 (L18924); ZMM1 (X81200); ZMM23 (AJ430637); EpM ADS20 (KJ002745); EpMADS21 (KJ002746); EpMADS22 (KJ002747); DcOAG1 (DQ119840); DthyrAG1 (DQ0 17702); CeMADS1 (GU123626); CeMADS2 (GU123627); AcoAG (Aco009993); AvAG1 (BAD18011.1); HAG1 (AAD19360.2); MaAG-1 (GSMUA_Achr10G21480_001); MaAG-2 (GSMUA_Achr10G14160_001); MaAG-3 (GSM UA_Achr5T06590_001); MaAG-4 (GSMUA_Achr6G14 760_001); PeMADS1 and PeMADS24 were obtained from the online OrchidBase website (http://orchidbase.itps.ncku. edu.tw/). Gene sequences cloned in this study have been deposited into Genbank database with the accession numbers MG021184 and MG021185. 


\section{Acknowledgements}

The authors thank Prof. Zhongjian Liu and Dr. Guogiang Zhang (National Orchid Conservation Center of China) for providing the Cymbidium varieties; Miss Maria João Ferreira (Faculdade de Ciências da Universidade do Porto) for her constructive suggestions on manuscript revision. The authors are also grateful to the anonymous reviewers for their encouraging and valuable comments on the manuscript. This work was supported by the Ministry of Science and Technology of the People's Republic of China (Grant No. 2013BAD01B0702)

\section{Conflict of interest}

The authors declare that they have no conflict of interest.

Supplementary Information accompanies this paper at (https://doi.org/ 10.1038/s41438-018-0052-z).

Received: 11 November 2017 Revised: 8 May 2018 Accepted: 17 May 2018 Published online: 01 September 2018

\section{References}

1. Li, X. et al. Genetic diversity, population structure, pollen morphology and cross-compatibility among Chinese Cymbidiums. Plant Breed. 133, 145 (2014).

2. Liu, Z., Chen, S., Ru, Z. \& Chen, L. The Genus Cymbidium in China. (Science Press, Beijing, China, 2006)

3. Duttke, S., Zoulias, N. \& Kim, M. Mutant flower morphologies in the wind orchid, a novel orchid model species. Plant Physiol. 158, 1542 (2012).

4. Schmidt, R. J. et al. Identification and molecular characterization of ZAG1, the maize homolog of the Arabidopsis floral homeotic gene AGAMOUS. Plant Cell 5, 729 (1993)

5. Yamaguchi, T. et al. Functional diversification of the two C-class MADS box genes OSMADS3 and OSMADS58 in Oryza sativa. Plant Cell 18, 15 (2006).

6. Dreni, L. \& Kater, M. M. MADS reloaded: evolution of the AGAMOUS subfamily genes. New Phytol. 201, 717 (2014).

7. Yanofsky, M. F. et al. The protein encoded by the Arabidopsis homeotic gene AGAMOUS resembles transcription factors. Nature 346, 35 (1990).

8. Drews, G. N., Bowman, J. L. \& Meyerowitz, E. M. Negative regulation of the Arabidopsis homeotic gene AGAMOUS by the APETALA2 product. Cell 65, 991 (1991).

9. Bowman, J. L., Smyth, D. R. \& Meyerowitz, E. M. Genetic interactions among floral homeotic genes of Arabidopsis. Development 112, 1 (1991).

10. Kramer, E. M., Jaramillo, M. A. \& Di Stilio, V. S. Patterns of gene duplication and functional evolution during the diversification of the AGAMOUS subfamily of MADS box genes in angiosperms. Genetics 166, 1011 (2004).

11. Heijmans, K. et al. Redefining $C$ and D in the petunia ABC. Plant Cell 24, 2305 (2012).

12. Davies, B. et al. PLENA and FARINELLI: redundancy and regulatory interactions between two Antirrhinum MADS-box factors controlling flower development. Embo J. 18, 4023 (1999).

13. Bowman, J. L., Smyth, D. R. \& Meyerowitz, E. M. Genes directing flower development in Arabidopsis. Plant Cell 1, 37 (1989).

14. Huang, Z. et al. APETALA2 antagonizes the transcriptional activity of AGAMOUS in regulating floral stem cells in Arabidopsis thaliana. New Phytol. 215, 1197 (2017).

15. Pinyopich, A. et al. Assessing the redundancy of MADS-box genes during carpel and ovule development. Nature 424, 85 (2003).

16. Causier, B. et al. Evolution in action: following function in duplicated floral homeotic genes. Curr. Biol. 15, 1508 (2005).

17. Dreni, L. et al. Functional analysis of all AGAMOUS subfamily members in rice reveals their roles in reproductive organ identity determination and meristem determinacy. Plant Cell 23, 2850 (2011).

18. Mena, M. et al. Diversification of C-function activity in maize flower development. Science 274, 1537 (1996).

19. Theißen, G., Strater, T., Fischer, A. \& Saedler, H. Structural characterization, chromosomal localization and phylogenetic evaluation of two pairs of AGAMOUS-like MADS-box genes from maize. Gene 156, 155 (1995).
20. Ambrose, B. A. et al. Molecular and genetic analyses of the silky1 gene reveal conservation in floral organ specification between eudicots and monocots. Mol. Cell 5, 569 (2000).

21. Lin, C. et al. Transcriptome-wide analysis of the MADS-box gene family in the orchid Enycina pusilla. Plant Biotechnol. J. 14, 284 (2016).

22. Salemme, M., Sica, M., Gaudio, L. \& Aceto, S. The OitaAG and OitaSTK genes of the orchid Orchis italica: a comparative analysis with other C- and D-class MADS-box genes. Mol. Biol. Rep. 40, 3523 (2013).

23. $\mathrm{Xu}$, Y. et al. Floral organ identity genes in the orchid Dendrobium crumenatum Plant J. 46, 54 (2006).

24. Wang, S. et al. Duplicated C-Class MADS-box genes reveal distinct roles in gynostemium development in Cymbidium ensifolium (Orchidaceae). Plant Cell Physiol. 52, 563 (2011).

25. Hsu, H. F. et al. C/D class MADS-box genes from two monocots, orchid (OncidiumGower Ramsey) and lily (Lilium longiflorum), exhibit different effects on floral transition and formation in Arabidopsis thaliana. Plant Cell Physiol. 51, 1029 (2010).

26. Chen, Y. et al. C- and D-class MADS-box genes from Phalaenopsis equestris (Orchidaceae) display functions in gynostemium and ovule development. Plant Cell Physiol. 53, 1053 (2012)

27. Almeida, A. M., Yockteng, R., Otoni, W. C. \& Specht, C. D. Positive selection on the $\mathrm{K}$ domain of the AGAMOUS protein in the Zingiberales suggests a mechanism for the evolution of androecial morphology. Evodevo 6, 7 (2015).

28. Zahn, L. M. et al. Conservation and divergence in the AGAMOUS subfamily of MADS-box genes: evidence of independent sub- and neofunctionalization events. Evol. Dev. 8, 30 (2006).

29. Liljegren, S. J. et al. SHATTERPROOF MADS-box genes control seed dispersal in Arabidopsis. Nature 404, 766 (2000).

30. Gaut, B. S. \& Doebley, J. F. DNA sequence evidence for the segmental allotetraploid origin of maize. Proc. Natl Acad. Sci. USA 94, 6809 (1997).

31. Pelaz, S. et al. B and C floral organ identity functions require SEPALLATA MADSbox genes. Nature 405, 200 (2000).

32. Coen, E. S. \& Meyerowitz, E. M. The war of the whorls: genetic interactions controlling flower development. Nature 353, 31 (1991).

33. Mondragón-Palomino, M. \& Theißen, G. Conserved differential expression of paralogous DEFICIENS- and GLOBOSA-like MADS-box genes in the flowers of Orchidaceae: refining the 'orchid code'. Plant J. 66, 1008 (2011).

34. Aceto, S. \& Gaudio, L. The MADS and the beauty: genes involved in the development of orchid flowers. Curr. Genom. 12, 342 (2011).

35. Chang, Y. Y. et al. Characterization of the possible roles for B class MADS box genes in regulation of perianth formation in orchid. Plant Physiol. 152, 837 (2010).

36. Kaufmann, $K$ et al. Target genes of the MADS transcription factor SEPALLATA3: integration of developmental and hormonal pathways in the Arabidopsis flower. PLoS Biol. 7, e1000090 (2009).

37. Darwin, C. On the Various Contrivances by Which British and Foreign Orchids are Fertilised by Insects. (John Murray, London, UK, 1862).

38. Kurzweil, H. Developmental studies in orchid flowers I: epidendroid and vandoid species. Nord J. Bot. 7, 427 (1987).

39. Kurzweil, H. Developmental studies in orchid flowers III: Neottioid species, Nord J. Bot. 8, 271 (1988).

40. Kurzweil, H. Developmental studies in orchid flowers II: Orchidoid species Nord J. Bot. 7, 443 (1987).

41. Lee, S. et al. Establishment of an Agrobacterium-mediated genetic transformation procedure for the experimental model orchid Enycina pusilla. Plant Cell Tiss. Org. 120, 211 (2015).

42. Tamura, K. et al. MEGA6: molecular evolutionary genetics analysis version 6.0. Mol. Biol. Evol. 30, 2725 (2013).

43. Su, S. et al. The CYCLOIDEA-RADIALIS module regulates petal shape and pigmentation, leading to bilateral corolla symmetry in Torenia fournieri (Linderniaceae). New Phytol. 215, 1582 (2017).

44. Zhu, G. et al. Transcriptome characterization of Cymbidium sinense 'Dharma' using 454 pyrosequencing and its application in the identification of genes associated with leaf color variation. Plos One 10, e128592 (2015).

45. Coen, E. S. et al. Floricaula: a homeotic gene required for flower development in Antirrhinum majus. Cell 63, 1311 (1990).

46. Clough, S. J. \& Bent, A. F. Floral dip: a simplified method for Agrobacteriummediated transformation of Arabidopsis thaliana. Plant J. 16, 735 (1998). 